\title{
Los humedales costeros del Pacífico sudamericano: ¿qué está sucediendo?
}

Wetlands of the South

American Pacific Coast: ¿What's going on?

Héctor Aponte ${ }^{1,2 * \odot}$ y Elier Tabilo ${ }^{\circledR}$

1 Universidad Científica del Sur. Lima, Perú. Coastal Ecosystems of Peru Research Group.

2 Centro Neotropical de Entrenamiento en Humedales. La Serena, Chile.

\section{SOUTH} SUSTAINABILITY

Citar como: Aponte, H. y Tabilo, E. (2021). «Los humedales costeros del Pacífico sudamericano: ¿qué está sucediendo?». South Sustainability 2(2), e034.

doi: https://doi.org/10.21142/SS-02022021-ed002

\section{(c) (1)}

(c) Los autores, 2021. Published by Universidad Científica del Sur (Lima, Perú)

${ }^{*} E$-mail de correspondencia: haponte@cientifica.edu.pe
La conservación de los humedales ha sido solicitada por diferentes instituciones a nivel mundial. En los últimos años se ha resaltado el importante valor de estos espacios con propuestas para el reconocimiento de sus derechos intrínsecos (Davies et al., 2020); también se publicaron estudios que muestran los beneficios económicos que obtenemos, debido a los servicios ecosistémicos que brindan a la sociedad (Davidson et al., 2019). En la costa del Pacífico sudamericano la comunidad científica realiza esfuerzos para documentar la diversidad que sobrevive en estos ambientes, con especial énfasis en su flora y fauna (Rivera et al., 2021). A ello se suma el esfuerzo de las organizaciones ciudadanas de conservación y el trabajo de autoridades gubernamentales. En ese marco se buscó y se sigue buscando generar espacios y herramientas que permitan la concertación de actores y la mejor toma de decisiones para estos ecosistemas.

Esto no significa que todo sea color de rosa. Algunos trabajos realizados en las ciudades costeras del Pacífico sudamericano muestran el deterioro que están sufriendo sus humedales. Por ejemplo, para los humedales costeros peruanos se cuenta con datos que describen la reducción de sus áreas (Flores et al., 2020), la contaminación de sus aguas (Rodríguez et al., 2017), el constante impacto que generan los incendios (Aponte et al., 2015), los cambios de los ciclos biogeoquímicos por actividades acuícolas (Pérez et al., 2020) y el arrojo de residuos sólidos que afecta considerablemente el aspecto de estas áreas (Aponte et al., 2020). En este país, se debe sumar la necesidad de continuar haciendo esfuerzos para mejorar las relaciones intersectoriales (León Sulca, 2020), procurando mantener la coherencia entre los aspectos técnicos y las decisiones que se toman para la gestión de estas áreas (evitando casos como el descrito en Ramirez y Aponte (2018) relacionado con la desafectación del humedal de Puerto Viejo). Son preocupantes los cambios de zonificación, construcción y drenaje que están ocurriendo en humedales costeros de Lima (como Santa Rosa, Quilcay y Ventanilla), muchas veces con el apoyo de nuestras autoridades.

La situación en Ecuador y Chile no es muy diferente. En Ecuador existe una alta presión en la zona costera del país por actividades productivas como las salineras y camaroneras, que provocan cambio en el uso del suelo en amplias zonas de manglar, lo que contribuye a una reducción significativa de los bosques de mangle y la contaminación de sus aguas y zonas costeras (por la cría y engorda de camarones), a lo que se suma al avance del desarrollo urbano. En Chile, la urbanización provoca efectos igualmente importantes en la calidad ambiental de las zonas costeras del país. Por ejemplo, alteraciones como el drenaje de humedales y las canalizaciones de cursos de agua llevan asociados eventos de contaminación 
(crónicos o en pulsos) con aguas servidas, aguas de riego con altos niveles de agroquímicos, e incluso se asocian a accidentes industriales (minería), eventos catastróficos por inundaciones, crecidas de ríos que colapsan depósitos de relaves dentro de la cuenca (Oyarzun et al., 2004; Román y Valdovinos, 2000). Estudios más recientes como Tabilo et al. (2016) describen cómo los centros poblados, desarrollo inmobiliario costero y actividades económicas como la minería y agricultura amenazan la integridad de los humedales costeros del centro norte de Chile.

Frente a ello y ante la creciente generación de información científica sobre estos ecosistemas es que nos preguntamos qué está pasando. ¿En qué momento se desconectó el conocimiento científico de la toma de decisiones? El Panel Intergubernamental sobre Cambio Climático nos dice claramente que no podemos dar ningún paso en falso (pueden revisar el editorial de nuestro número anterior, donde compartimos una reflexión al respecto); sin embargo, se continúan realizando actividades que merman la salud de los humedales y de otros ecosistemas de nuestro planeta. El ser humano es una especie que llevó al colapso muchos espacios de la Tierra, colapso que les jugó en contra a estas mismas civilizaciones, al obligarlas a dejar el lugar en donde viven (Diamond, 2012). Esperamos que ese no sea el caso de las poblaciones que dependen de los humedales, en especial en la zona costera desértica del Pacífico sudamericano. Estamos a tiempo de tomar decisiones correctas por nuestro bienestar y el de las futuras generaciones.

\section{Conflictos de interés}

Los autores son investigadores de humedales costeros y pertenecen a instituciones (ONG y universidades) que tienen como especial interés la protección de estos ecosistemas.

\section{Referencias bibliográficas}

Aponte, H., Gonzales, S. y Gomez, A. (2020). «Impulsores de cambio en los humedales de América Latina: el caso de los humedales costeros de Lima». South Sustainability, 1, e023 (1-5). Disponible en: https://doi.org/10.21142/SS-0102-2020-023

Aponte, H., Ramírez, D., Lértora, G., Vargas, R., Gil, F., Carazas, N. y Liviac, R. (2015). «Incendios en los humedales de la costa central del Perú: una amenaza frecuente». Científica, 12(1), pp. 70-81.

Davidson, N. C., Dam, A. A. van, Finlayson, C. M. y Mclnnes, R. J. (2019). «Worth of wetlands: revised global monetary values of coastal and inland wetland ecosystem services». Marine and Freshwater Research, 70, pp. 1189-1194. Disponible en: https://doi. org/10.1071/MF18391

Davies, G. T., Finlayson, C. M., Pritchard, D. E., Davidson, N. C., Gardner, R. C., Moomaw, W. R., Okuno, E., Whitacre, J. C., Davies, G. T., Finlayson, C. M., Pritchard, D. E., Davidson, N. C., Gardner, R. C., Moomaw, W. R., Okuno, E. y Whitacre, J. C. (2020). «Towards a Universal Declaration of the Rights of Wetlands». Marine and Freshwater Research, 72, pp. 593-600. Disponible en: https://doi. org/10.1071/MF20219

Diamond, J. (2012). Colapso. Por qué unas sociedades perduran y otras

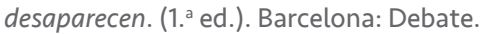

Flores, N., Castro, I., Aponte, H., Flores, N., Castro, I. y Aponte, H. (2020). «Evaluación de las unidades de vegetación en Los Pantanos de Villa (Lima, Perú) mediante sistemas de información geográfica y teledetección». Arnaldoa, 27, pp. 303-321. Disponible en: https://doi. org/10.22497/arnaldoa.271.27119

León Sulca, G. M. (2020). Gobernanza ambiental y conservación: las gestiones del SERNANP y PROHVILLA en el Refugio de Vida Silvestre Los Pantanos de Villa. Revista Argumentos, 1, pp. 119-124. Disponible en: https://doi.org/10.46476/ra.vi1.20

Oyarzun, R., Lillo, J., Higueras, P., Oyarzún, J. y Maturana, H. (2004). «Strong arsenic enrichment in sediments from the Elqui watershed, Northern Chile: industrial (gold mining at El Indio-Tambo district) vs. geologic processes». Journal of Geochemical Exploration, 84, pp. 53-64. Disponible en: https://doi.org/10.1016/j.gexplo.2004.03.002

Pérez, A., Machado, W., Gutiérrez, D., Saldarriaga, M. S. y Sanders, C. J. (2020). «Shrimp farming influence on carbon and nutrient accumulation within Peruvian mangroves sediments». Estuarine, Coastal and Shelf Science, 243, 106879. Disponible en: https://doi. org/10.1016/j.ecss.2020.106879

Ramirez, D. W. y Aponte, H. (2018). «Por qué los Humedales de Puerto Viejo perdieron su protección legal: analizando los motivos». Revista Peruana de Biología, 25, pp. 049-054.

Rivera, G., Gonzales, S. y Aponte, H. (2021). Wetlands of the South American Pacific coast: a bibliometric analysis. Wetlands Ecol Manage. Disponible en: https://doi.org/10.1007/s11273-021-09830-8

Rodríguez, R., Retamozo-Chavez, R., Aponte, H. y Valdivia, E. (2017). «Evaluación microbiológica de un cuerpo de agua del ACR Humedales de Ventanilla (Callao, Perú) y su importancia para la salud pública local». Ecología Aplicada, 16, pp. 15-21. Disponible en: https://doi.org/10.21704/rea.v16i1.899

Román, H. y Valdovinos, C. (2000). «Una aproximación al estudio integral de la contaminación del río Loa, II Región, Chile: Período marzo 1997-febrero 2000». Primer simposio sobre medioambiente «Gestión ambiental e investigación en metales pesados en el norte de Chile». Antofagasta: Centro Regional de Estudios Ambientales (CREA) de la Universidad de Antofagasta.

Tabilo, E., Burmeister, J., Chavez, C. y Zuckler, C. (2016). «Humedales y aves migratorias en la costa árida del Pacífico sudamericano. Etapa 1». Centro Neotropical de Entrenamiento en Humedales y Manfred Hermsen Stiftung. 